\title{
RECIPROCAL ALTERED EXPRESSION OF E-CADHERIN AND P-CADHERIN IN MUCOUS MEMBRANE PEMPHIGOID
}

\author{
A. SANTARELLI I,, O. DI FEDE ${ }^{3}$, F. CARINCI ${ }^{4}, \mathrm{C}^{1}$. RUBINI ${ }^{5}$, \\ G. GANZETTI ${ }^{6}$, D. PERRONE ${ }^{1}$, O. SIMONETTI ${ }^{6}$, A. OFFIDANI ${ }^{6}, \mathrm{M}^{2}$ RE ${ }^{2}$, D. PERRONE ${ }^{1}$, \\ G. PANNONE ${ }^{1}$, L. LO MUZIO ${ }^{1,3}$ \\ ${ }^{1}$ Department of Surgery, University of Foggia, Foggia, Italy \\ ${ }^{2}$ Department of Specialistic Clinic and Odontostomatological Sciences, \\ Polythecnic University of Marche, Ancona, Italy \\ ${ }^{3}$ Department of Oral Sciences, Sector of Oral Medicine “V. Margiotta”, University of Palermo, \\ Palermo, Italy \\ ${ }^{4}$ Department of D.M.C.C.C., Section of Maxillofacial and Plastic Surgery, University of Ferrara, Ferrara, Italy \\ ${ }^{5}$ Institute of Pathology, Polytechnic University of Marche, Ancona, Italy \\ ${ }^{6}$ Clinic of Dermatology, Polytechnic University of Marche, Ancona, Italiy
}

E- and P- cadherins are involved in the selective adhesion of epidermal cells. To gain insight into the role of cadherins on the acantholysis of keratinocytes and further investigate the pathogenesis of Mucous Membrane Pemphigoid, we examined the expression of P-cadherin and E-cadherin, in normal human oral mucosa, lesional and peri-lesional mucosa in MMP. Twenty-nine samples from paraffin-embedded specimens of MMP were used for the study. Five specimens of healthy oral mucosa were evaluated as control group. To evaluate the E- and P-Cadherin expression, a mean percentage of positive cells was determined from the percentage of positive cells derived from the analysis of 100 cells in ten random areas at $\mathbf{x 4 0 0}$ magnification. It was observed that E-cadherin was weakly and discontinuously expressed on the epithelial layers of pemphigoid mucosa, while it was intensively expressed on all keratinocytes in normal human skin. In contrast, P-cadherin was strongly expressed throughout the entire epidermal layer in MMP samples, although its expression is restricted to the basal cell layer in normal human skin. Statistical analyses showed that the percentage of E-cadherin positive cells in the epithelium of pemphigoid cases was significantly decreased compared with that in normal human mucosa. There was a significant increase in the percentage of P-cadherin positive cells in the epithelial layers of MMP compared with normal human mucosa. The present study showed that there is downregulation of E-cadherin expression and upregulation of P-cadherin expression in MMP mucosa, which may be involved in the pathogenesis of MMP.

Autoimmune subepidermal blistering disorders are a large family of skin/mucous diseases including, among others, mucous membrane pemphigoid (MMP) (previous named cicatricial pemphigoid)(1). In vivo linear deposition of immune deposits (immunoglobulins, complement or both) along the basement membrane zone (BMZ) was the common feature that characterize pemphigoid (2). However, while bullous pemphigoid (BP) generally affect the skin and have only minor oral involvement, MMP mainly involves the mucous membranes, most frequently the oral and ocular mucosa (3).

Among the classical cadherins, E- (epithelial) and P- (placental) cadherins are involved in the selective adhesion of epidermal cells. E-cadherin is expressed on the cell surfaces of all epidermal layers, whereas Pcadherin is expressed only on the surfaces of the basal and most immediate suprabasal cells of the epidermis (4-6).

To the best of our knowledge, the expression of Eand P-cadherin in MMP lesions has not previously been reported. Thus, to gain insight into the role of cadherins

Key words: Blistering disease, E-cadherin, Mucous Membrane Pemphigoid, P-cadherin

Corresponding author: Prof. Francesco Carinci, M.D

Department of D.M.C.C.C. Section of Maxillofacial and Plastic Surgery

University of Ferrara Corso Giovecca 203

44100 Ferrara ITALY

0394-6320 (2011)

E-mail: crc@unife.it

Web: www.carinci.org

Phone: +39.0532.455874; Fax: +39.0532 .455582$

$103(\mathrm{~S})$

Copyright $(\mathrm{C}$ by BIOLIFE, s.a.s.

This publication and/or article is for individual use only and may not be further reproduced without written permission from the copyright holder. Unauthorized reproduction may result in financial and other penalties 
on the keratinocytes pathology and further investigate the pathogenesis of MMP, we examined the expression of P-cadherin, as well as E-cadherin, in normal human oral mucosa, lesional and peri-lesional mucosa in MMP.

\section{MATERIALS AND METHODS}

\section{Selection of cases}

The study population consisted of 11 men and 18 women with a mean age of 66 years (age range 41-84). None of patients had been previously treated with topical or systemic steroid therapy.

Twenty-nine samples from paraffin-embedded specimens of mucous membrane pemphigoid (MMP) were used for the study. Specimens were fixed in 10\% neutral-buffered formalin.

The histological diagnosis was assessed on paraffin $\mathrm{H} \& \mathrm{E}$ - stained sections.

Five paraffin-embedded specimens of healthy oral mucosa were obtained from patients who had undergone routine oral surgical procedures, with the informed consent of the donors, and were evaluated as control group.

\section{Immunohistochemistry}

Four- $\mu \mathrm{m}$ serial sections from formalin-fixed, paraffinembedded blocks of MMP representative areas were cut for each case. Only sections containing sufficient epithelium to assess the antibody reactivity with 1000 cells were considered for this study.

Immunohistochemistry was then performed on the remaining sections mounted on poly-L-lysine-coated glass slides. Deparaffined and rehydrated sections were incubated for 30 minutes in $3 \% \mathrm{H}_{2} \mathrm{O}_{2} /$ methanol to quench endogenous peroxidase activity, and then rinsed for 20 minutes with phosphate-buffered saline (PBS) (Bio-Optica M107, Milan Italy). Non-specific protein binding was attenuated by incubation for 30 minutes with $5 \%$ horse serum in PBS. Specimens were incubated overnight with mouse monoclonal IgG antibody against P-cad and E-cad (Transduction Laboratories, Lexington, Kentucky, U.S.A.) used at a dilution of 1:300. The antibody was applied directly to the section and the slides were incubated overnight $\left(4^{\circ} \mathrm{C}\right)$. The sections were washed three times with PBS at room temperature. Immune complexes were subsequently treated with the secondary biotinilated antibody and then detected by streptavidin peroxidase, both incubated for 30 minutes at room temperature (Vectastain $\mathrm{ABC}$ kit, Vector Laboratories, Burlingame, CA, USA). After rinsing with 3 changes of PBS the immunoreactivity was visualized by development for 2 minutes with $0,1 \% 3,3$ '-diaminobenzidine and $0,02 \%$ hydrogen peroxide (DAB substrate kit, Vector Laboratories Burlingame Calif.). Sections were counterstained with Mayer's haematoxylin, mounted with permanent mounting medium and examined by light microscopy.

Positive controls consisted of tissue specimen sections of normal skin with known antigenic reactivity. A negative control was performed in all cases by substituting the primary antibodies with non-immune serum. Negative controls in all instances resulted in a negative immunoreactivity for E- and P-Cadherin. To evaluate the E- and P-Cadherin expression, a mean percentage of positive cells was determined from the percentage of positive cells derived from the analysis of 100 cells in ten random areas at $\mathrm{x} 400$ magnification. The positivity for E- and P-Cadherin was evaluated by an expert pathologist of the field.

Data were analysed using GraphPad Prism software version 5.00 for Windows (GraphPad Software, San Diego California USA, www.graphpad.com). Significant differences $(\mathrm{P}<0.05)$ between groups were determined using one-way analysis of variance (ANOVA). A p value of less than 0.05 was accepted as statistically significant.

\section{RESULTS}

\section{E- and P-cadherin expression in normal oral mucosa}

Both E- and P- cadherin showed Normal human oral mucous epithelium had a basal and para-basal pattern of P-cadherin expression (Fig. 1 A). The labeling was only membranous, as well as for E-cadherin. Unlike, E-cadherin was expressed throughout the whole oral epithelium. Indeed, also keratinocytes of supra-basal layers were immunolabelled by anti-E-cadherin antibody (Fig. 1 B). Thus, normal epithelium included around 20\% of stained cells for P-cadherin and 70\% for E-cadherin.

\section{Downregulation of E-cadherin expression in MMP oral тисова}

In MMP samples, the staining pattern of E-cadherin differed from that seen in normal mucosa. Indeed, it was observed that E-cadherin was weakly and discontinuously expressed throughout the entire epidermal layer of pemphigoid mucosa (Fig $1 \mathrm{C}$ ).

\section{Upregulation of P-cadherin expression in MMP oral mисова}

A distinctively different immunostaining pattern of P-cadherin was found between the lesional, the perilesional and the normal human mucosa. In normal human mucosa, the expression of P-cadherin was limited to the basal cell layer as previously demonstrated. In contrast, in MMP cases the stronger positive immunostaining of P-cadherin was observed not only in the basal cell layer, but also in the suprabasal layers in lesional mucosa (Fig. $1 \mathrm{D})$.

\section{Statistical analyses}

Statistical analyses showed that the percentage of Ecadherin positive cells in the epithelium of pemphigoid cases was significantly decreased compared with normal and peri-lesional human mucosa $(p<0.05)$ (Fig. 2 A). On the contrary, there was a significant increase in the percentage of P-cadherin positive cells in the epithelial layers of MMP compared with normal and peri-lesional human mucosa $(\mathrm{p}<0.05)$ (Fig. 2 B). 


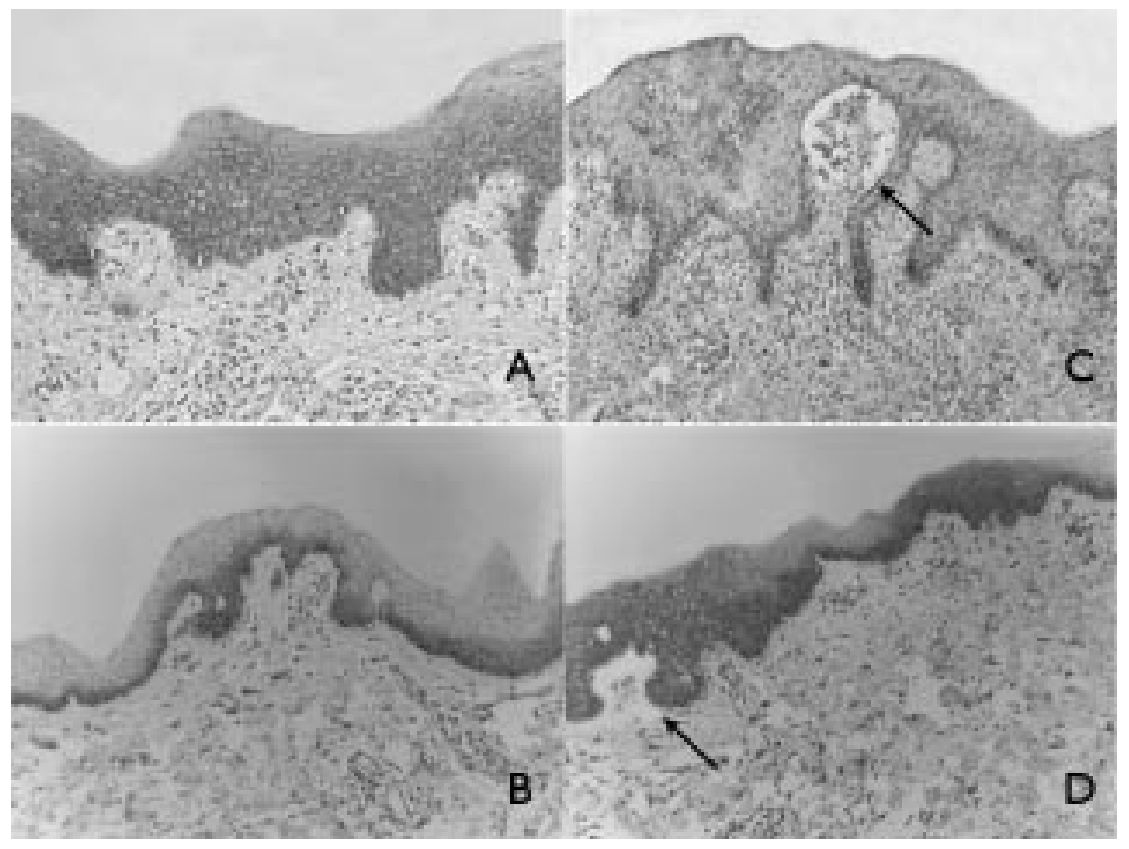

Fig.1. Immunohistochemical staining for $E$ - and P-cadherin expression in normal human mucosa $(A, B)$ and blister mucosa $(C$, D). Normal mucosa: membranous positivity for E-cadherin, from basal to spinous layer. (E-cadherin, $x$ 160) (A); Normal mucosa: membranous positivity for P-cadherin, basal layer. (P-cadherin, $x$ 100) (B); Pemphigoid: sub-epithelial blister (black arrow) negative for E-caherin expression. (E-cadherin, x160) (C); Pemphigoid: sub-epithelial blister (black arrow) positivity for P-cadherin expression, from basal to spinous layer. (P-cadherin, x.100) (D)
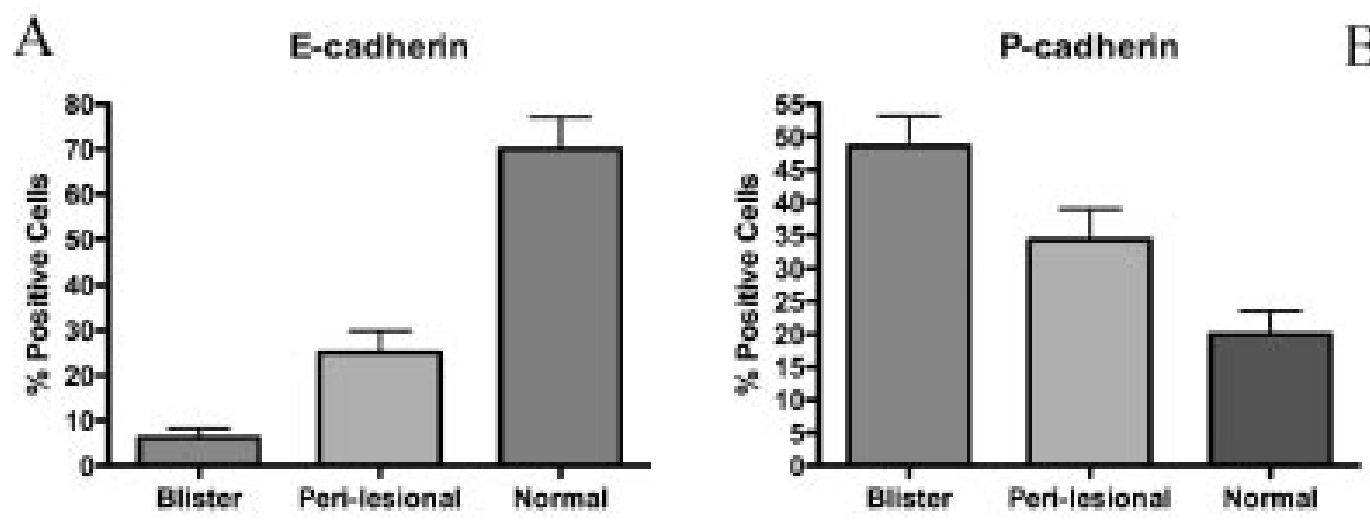

Fig. 2. Percentage of E-cadherin positive cells in the epithelium of pemphigoid cases compared with normal and peri-lesional human mucosa (A) and percentage of P-cadherin positive cells in the epithelial layers of MMP compared with normal and peri-lesional human mucosa (B).

\section{DISCUSSION}

E- and P-cadherin are the major classical cadherins in the epidermis. E-cadherin is expressed on almost all epidermal cells and thought to be the main cadherin type responsible for intercellular adhesion $(6,7)$. P-cadherin was originally identified in mouse placental tissue and appeared to act as a connector between the embryo and the uterus $(6,8)$. In normal epidermis, P-cadherin is restricted in the cell surface of the basal layer and considered to be associated with the maintenance of proliferation and differentiation of epidermal keratinocytes $(4,5)$. The inhibition of $\mathrm{P}$-cadherin resulted in a stronger perturbation of skin morphogenesis than that of E-cadherin (5).

In MMP samples, the staining patterns of both cadherins differed from those seen in normal mucosa. 
The present study demonstrated that the expression of Ecadherin was significantly downregulated in peri-lesional and much more in lesional mucosa of oral MMP, whereas P-cadherin expression was significantly upregulated in peri-lesional and in lesional mucosa.

To the best of our knowledge, this is the first report investigating the reciprocal expression of these two cadherins in mucous pemphigoid. However, present findings were concordant with previous results on cadherin expression in blistering diseases. Indeed, the upregulation of P-cadherin expression seems to be a common event in autoimmune blistering diseases like pemphigus vulgaris, pemphigus foliaceus, bullous pemphigoid and dermatitis herpetiformis (9) as well as in inherited acantholytic disease like Darier's and Hailey-Hailey diseases (10) and non-blistering diseases as psoriasis vulgaris (11).

The actual knowledge in this field allows only to rise some hypotheses that could explain the altered cadherin expression. The involvement of P-cadherin upregulation in the pathogenesis of both autoimmune and inherited blistering diseases has been suggested (10).

However, it does not seem likely. Indeed, according to Kovac, the upregulation of P-cadherin is not playing a causal role in the pathophysiology, but it might be a compensatory consequence (9). Since in vivo synergism between Pcadherin and desmoglein-3 has been demonstrated, suggesting a cooperative/compensatory effect of these adhesion molecules (12), a similar mechanism could be postulated between E- and P-cadherin. This theory could justify the findings of the present study as well as those of recent reports. It could also explain why in MMP and other blistering disease P-cadherin expression show a diffuse distribution across all epithelial layers, resembling the distribution of E-cadherin in normal skin/mucosa.

In conclusion, the present study demonstrated for the first time that downregulation of E-cadherin expression and upregulation of P-cadherin expression occurred in the lesional and peri-lesional oral mucosa of MMP. Further studies are necessary to investigate the role that the reciprocal altered expression of E- and P-cadherin could exert in MMP. Clarifying the mechanism of the altered expression of E- and P-cadherin pemphigoid mucosa will provide new insight regarding not only the pathogenesis of MMP but also the biological roles of E- and P-cadherin.

\section{REFERENCES}

1. Verdolini R, Cerio R. Autoimmune subepidermal bullous skin diseases: the impact of recent findings for the dermatopathologist. Virchows Arch 2003; 443:184-93.

2. Beutner EH, Jordon RE, Chorzelski TP. The immunopathology of pemphigus and bullous pemphigoid. J Invest Dermatol 1968; 51:63-80.

3. Liu HN, Su WP, Rogers RS, 3rd. Clinical variants of pemphigoid. Int J Dermatol 1986; 25:17-27.

4. Fujita M, Furukawa F, Fujii K, Horiguchi Y, Takeichi M, Imamura S. Expression of cadherin cell adhesion molecules during human skin development: morphogenesis of epidermis, hair follicles and eccrine sweat ducts. Arch Dermatol Res 1992; 284:159-66.

5. Furukawa F, Takigawa M, Matsuyoshi N, Shirahama S, Wakita H, Fujita M, Horiguchi Y, Imamura S. Cadherins in cutaneous biology. J Dermatol 1994; 21:802-13.

6. Shimoyama Y, Hirohashi S, Hirano S, Noguchi M, Shimosato Y, Takeichi M, Abe O. Cadherin cell-adhesion molecules in human epithelial tissues and carcinomas. Cancer Res 1989; 49:2128-33.

7. Wheelock MJ, Jensen PJ. Regulation of keratinocyte intercellular junction organization and epidermal morphogenesis by E-cadherin. J Cell Biol 1992; 117:41525.

8. Nose A, Takeichi M. A novel cadherin cell adhesion molecule: its expression patterns associated with implantation and organogenesis of mouse embryos. J Cell Biol 1986; 103:2649-58.

9. Kovacs A, Schmidt E, Begany A, Hunyadi J, Szegedi A. Immunohistochemical examination of P-cadherin in bullous and acantholytic skin diseases. Acta Derm Venereol 2004; 84:116-19.

10. Hakuno M, Akiyama M, Shimizu H, Wheelock MJ, Nishikawa T. Upregulation of P-cadherin expression in the lesional skin of pemphigus, Hailey-Hailey disease and Darier's disease. J Cutan Pathol 2001; 28:277-81.

11. Zhou S, Matsuyoshi N, Takeuchi T, Ohtsuki Y, Miyachi Y. Reciprocal altered expression of T-cadherin and Pcadherin in psoriasis vulgaris. Br J Dermatol 2003; 149: 268-73.

12. Lenox JM, Koch PJ, Mahoney MG, Lieberman M, Stanley JR, Radice GL. Postnatal Lethality of P-Cadherin/ Desmoglein 3 Double Knockout Mice: Demonstration of a Cooperative Effect of these Cell Adhesion Molecules in Tissue Homeostasis of Stratified Squamous Epithelia. J Invest Dermatol 2000; 114:948-52. 